\title{
Erratum to: Historical development of theories of the electrochemical double layer
}

\author{
B. B. Damaskin • O. A. Petrii
}

Published online: 1 November 2011

(C) Springer-Verlag 2011

Erratum to: J Solid State Electrochem (2011) 15:1317-1334

DOI 10.1007/s10008-011-1294-y

The authors regret errors in Eq. 31 and in the caption of Fig. 4. The correct Eq. 31 is

$K_{02}=K_{02}^{0}(1-\theta)+K_{02}^{1} \theta$

The correct caption of Fig. 4 is:

Fig. 4 Differential capacitance curves in system (C): symbols - calculations according to the model of three parallel capacitors with subsequent allowance for the diffuse layer capacitance; lines - calculations by the $2 \mathrm{PC}$ model; numbers - the n-butanol concentrations (in M).

The online version of the original article can be found at http://dx.doi. org/10.1007/s10008-011-1294-y.

B. B. Damaskin · O. A. Petrii $(\bowtie)$

Lomonosov Moscow State University,

Leninskie Gory, 1-str.3,

Moscow 119992, Russia

e-mail: petrii@elch.chem.msu.ru 